\title{
shRNA-mediated silencing of the RFC3 gene suppresses hepatocellular carcinoma cell proliferation
}

\author{
ZHICHENG YAO*, KUNPENG HU*, HE HUANG, SHILEI XU, QINGLIANG WANG, \\ PENG ZHANG, PEISHENG YANG and BO LIU \\ Department of General Surgery, The Third Affiliated Hospital, Sun Yat-Sen University, \\ Guangzhou, Guangdong 510530, P.R. China
}

Received May 14, 2015; Accepted September 9, 2015

DOI: $10.3892 /$ ijmm.2015.2350

\begin{abstract}
Hepatocellular carcinoma (HCC) is one of the most common and lethal malignancies worldwide. Replication factor C (RFC) plays an important role in DNA replication and checkpoint control during the cell cycle. $\mathrm{RFC}$ is comprised of one large subunit [replication factor C, subunit 1 (RFC1)] and four small subunits [replication factor C, subunits 2-5 (RFC2-5)]. The role of RFC3 in the development of $\mathrm{HCC}$ is, as of yet, not fully understood. In the present study, western blot analysis and reverse-transcriptionquantitative PCR were used to measure the expression levels of replication factor $\mathrm{C}$, subunit 3 (RFC3) in HCC tissues and HCC cells. Lentivirus-mediated RFC3-specific short hairpin RNA (shRNA) was used to knock down the expression of RFC3 in HCC cells in order to examine the effects of RFC3 on HCC cell proliferation and growth. Furthermore, the expression levels of cell cycle-related proteins were also measured in the HCC cells in which RFC3 was knocked down. Our results revealed that the expression level of RFC3 was markedly upregulated in the HCC tissues and cells. In addition, MTS and cell growth assays were used to determine the viability and proliferation of the HCC cells in which RFC3 was knocked down, and the results revealed that both cell viability and proliferation were effectively suppressed. The downregulation of RFC3 expression led to HCC cell cycle arrest in the $\mathrm{S}$ phase, partly by regulating the epression of cell cycle-related proteins, such as p21, p53, p57 and cyclin A. The results of the present study suggest that RFC3 plays an important role in the development of HCC, and may thus be a potential biological target in the treatment of HCC.
\end{abstract}

Correspondence to: Dr Bo Liu, Department of General Surgery, The Third Affiliated Hospital, Sun Yat-Sen University, 600 Tianhe Road, Tianhe, Guangzhou, Guangdong 510530, P.R. China E-mail: liubo150509@163.com

\section{${ }^{*}$ Co-first authors}

Key words: hepatocellular carcinoma, replication factor $\mathrm{C}$, cell growth, cell proliferation, cell cycle arrest, cell cycle-related proteins

\section{Introduction}

Hepatocellular carcinoma (HCC) is one of the most common and lethal malignancies worldwide $(1,2)$. An estimated 350,000 deaths from liver cancer occur worldwide each year (3). The highest liver cancer rates are to be found in East and Southeast Asia, and in Central and Western Africa; chronic hepatitis $\mathrm{B}$ virus (HBV) and $\mathrm{C}(\mathrm{HCV})$ infection are responsbile for approximately $75-80 \%$ of the HCC cases worldwide, particularly in Asian and African populations $(4,5)$.

The fidelity of DNA replication is generally considered an important characteristic of cancer progression and during the cell cycle. Dysfunctional DNA damage repair and checkpoints during the cell cycle process contribute to genomic instability. Replication factor C (RFC) is a heteropentameric primer-recognition protein complex involved in DNA replication, DNA damage repair and checkpoint control during cell cycle progression (6-10). The RFC complex functions to load proliferating cell nuclear antigen (PCNA), a ring-shaped homotrimer, onto DNA in an ATP-dependent manner in order to provide a sliding clamp for various proteins involved in DNA replication processes (11).

RFC is comprised of one large subunit [replication factor $\mathrm{C}$, subunit 1 (RFC1)] and four small subunits [replication factor $\mathrm{C}$, subunits 2-5 (RFC2-5)]. Of these subunits, replication factor C, subunit 3 (RFC3), a 38-kDa subunit, has been reported to be overexpressed in esophageal adenocarcinomas and ovarian carcinomas $(12,13)$. Moreover, RFC3 knockdown has been shown to result in the inhibition of cancer cell proliferation and growth $(12,14)$. The disruption of the RFC3-PCNA complex induced by 9-cis retinoic acid-activated retinoid $\mathrm{X}$ receptor $\alpha(\mathrm{RXR} \alpha)$ has been shown to inhibit the growth of cancer and embryonic cells and to arrest $\mathrm{S}$ phase entry (15). These findings suggest that RFC3 may be one of the most important cancer antigens. However, its role in the development of HCC remains unclear.

In this study, we found that RFC3 was overexpressed in $\mathrm{HCC}$ tissues and cells. Further investigations revealed that RFC3 is a critical factor in promoting the development of $\mathrm{HCC}$, as the silencing of RFC3 by shRNA led to cell cycle arrest. Our data provide new insight into the role of RFC3 in the development of HCC. 


\section{Materials and methods}

Tissue samples. Liver tumor tissue samples were obtained from 24 patients (age: mean 55, rage 40-68, gender: female 5 , male 19) who were diagnosed with HCC at the Third Affiliated Hospital, Sun Yat-Sen University, Guangzhou, China in 2012. A total of 24 human HCC tissues and 12 adjacent non-tumor tissue samples were examined in this study. For each case, tumor samples with matched adjacent non-tumor tissue samples were collected during surgical resection and frozen in liquid nitrogen and stored at $-80^{\circ} \mathrm{C}$. This study was approved by the Ethics Committee of Sun Yat-Sen University and all patients provided written informed consent prior to obtaining the samples.

Cell lines and culture. In this study, we used 1 human hepatocyte cell line (L02) and 5 HCC cell lines (HepG2, BEL-7402, Hep3B, SMMC-7721 and LM3), obtained from Shanghai Cell Bank (Chinese Academy of Science), to detect RFC3 expression. The cells were maintained in Dulbecco's modified Eagle's medium (DMEM) (Gibco BRL, Paisley, Scotland, UK) supplemented with $10 \%$ fetal calf serum, $100 \mathrm{IU} / \mathrm{ml}$ penicillin, $100 \mu \mathrm{g} / \mathrm{ml}$ streptomycin, and $2 \%$ L-glutamine (all from Biological Industries Israel Beit-Haemek Ltd. Kibbutz Beit-Haemek, Israel) at $37^{\circ} \mathrm{C}$ in an atmosphere with $5 \% \mathrm{CO}_{2}$.

$R N A$ isolation and RT-qPCR. Total RNA was extracted from the tissues and cells using TRIzol reagent (Invitrogen, Carlsbad, CA, USA) according to the instructions provided by the manufacturer. Reverse transcription was performed using a reverse transcription kit (Takara Bio, Dalian, China), and primers were designed as follows: RFC3 forward, 5'-GCC TGCAGAGTGCAACAATA-3' and reverse, 5'-TCAAGG AGCCTTTGTGGAGT-3'; and GAPDH forward, 5'-GAGT CAACGGATTTGGTCGT-3' and reverse, 5'-GACAAGCTT CCCGTTCTCAG-3'. Amplification reactions were performed in a $20 \mu 1$ volume of SYBR-Green PCR Master mix (from Takara Bio). All the reactions were performed in triplicate in a LightCycler Real-Time PCR system. The RFC3 mRNA expression levels were standardized to the GAPDH mRNA levels using the comparative $\mathrm{Ct}$ method. All experiments were performed at least 3 times.

Immunohistochemistry (IHC).IHC was performed as previously described (16). Briefly, the tumor sections were deparaffinized using xylene and rehydrated with graded ethyl alcohol, and a solution of $3 \%(\mathrm{v} / \mathrm{v}) \mathrm{H}_{2} \mathrm{O}_{2}$ was then added to halt the peroxidase activity. Antigen retrieval was performed by heating the tumor sections in $10 \mathrm{mM}$ sodium citrate buffer $(\mathrm{pH} 6.0)$ at $95-100^{\circ} \mathrm{C}$ for $20 \mathrm{~min}$. After being washed 3 times with phosphatebuffered saline (PBS; Sigma-Aldrich, St. Louis, MO, USA), the sections were blocked with $3 \%$ bovine serum albumin (BSA; Sigma-Aldrich) at room temperature for $1 \mathrm{~h}$, and this was followed by overnight incubation at $4{ }^{\circ} \mathrm{C}$ with RFC3 antibody (sc-390293; 1:100 dilution; Santa Cruz Biotechnology, Inc., CA, USA). After being washed 3 times with PBS, the sections were incubated at $37^{\circ} \mathrm{C}$ for $2 \mathrm{~h}$ with secondary antibodies. Finally, the sections were counterstained with hematoxylin.

Western blot analysis. The cells were harvested and then lysed in radioimmunoprecipitation assay (RIPA) buffer
[50 mM Tris- $\mathrm{HCl}(\mathrm{pH} 7.4), 150 \mathrm{mM} \mathrm{NaCl}, 1 \% \mathrm{NP}-40$, $0.25 \%$ Na-deoxycholate, $1 \mathrm{mM}$ EDTA and $1 \mathrm{mM} \mathrm{NaF}$, containing protease inhibitor cocktail (Sigma-Aldrich). The cell lysates were boiled for $5 \mathrm{~min}$ and refrigerated on ice, and this was followed by centrifugation at $10,000 \mathrm{x}$ g for $30 \mathrm{sec}$. Proteins were resolved by sodium dodecyl sulfate-polyacrylamide gel electrophoresis (SDS-PAGE) and electrotransferred onto polyvinylidene fluoride (PVDF) membranes. The membranes were blocked in 5\% non-fat dry milk and then probed with the primary antibodies against RFC3 (sc-390293; 1:500 dilution; Santa Cruz Biotechnology, Inc.) and p53 (ab31333; 1:500 dilution), p21 (ab7960; 1:200 dilution), p57 (ab75974; 1:500 dilution), cyclin A (ab137769; 1:1,000 dilution) and cyclin B1 (ab32053; 1:3,000 dilution) (all from Abcam, Cambridge, MA, USA). Subsequently, the membranes were washed twice with TBST and incubated with horseradish peroxidase-conjugated AffiniPure goat anti-mouse $\operatorname{IgG}(\mathrm{H}+\mathrm{L})$ (115-035-003; 1:5,000 dilution) or goat anti-rabbit IgG $(\mathrm{H}+\mathrm{L})$ (111-035-003; 1:5,000 dilution; both from Jackson ImmunoResearch, West Grove, PA, USA) secondary antibodies at room temperature for $1 \mathrm{~h}$. The membranes were washed another 3 times and then visualized using an ECL kit (Forevergen Biosciences Co., Ltd., Guangzhou, China).

Construction of shRFC3 lentivirus and gene silencing. The lentiviral vector, LV-008 (Forevergen Biosciences Co., Ltd.), expressing short hairpin RNA (shRNA) and containing the green fluorescent protein (GFP) gene was used as a reporter. The recombinant lentiviruses were designed to generate shRNA targeting the sequence of the RFC3 gene (5'-AAGTAACTACCACCTTGAAGTTA-3') and negative control (NC) (5'-TGGTTTACATGTCGACTAA-3'). The LV-008-shRFC3 plasmids were transfected into 293T cells (Shanghai Cell Bank, Chinese Academy of Science), together with the lentiviral packaging vectors, to generate the respective lentiviruses. Infection lentiviruses were collected at $72 \mathrm{~h}$ post-transfection, and the lentiviruses were concentrated by ultracentrifugation for $1.5 \mathrm{~h}$ at 25,000 rpm in an SW28 rotor (Bekcman Instruments Inc., Fullerton, CA, USA). For lentiviral infection, the SMMC-7721 cells were seeded in a 6-well plate at a density of 50,000 cells/well and infected with the lentiviruses in the presence of $5-10 \mu \mathrm{g} / \mathrm{ml}$ of polybrene. The cells in which RFC3 was knocked down were screened out with $2 \mu \mathrm{g} / \mathrm{ml}$ puromycin for 10-15 days. The knockdown efficiency was validated by RT-qPCR and western blot analysis on day 5 post-infection. Each experiment was performed in triplicate.

Colony formation assay. The cells were digested at the logarithmic growth phase and seeded into 6-well plates at density gradients of 50, 100 and 200 cells/well. Following 2 weeks of culture, the cells were washed and fixed with $4 \%$ paraformaldehyde for $30 \mathrm{~min}$ at room temperature, and then stained with crystal violet. The number of colonies was counted under a fluorescence microscope (BX-50; Olympus, Tokyo, Japan). Each experiment was performed in triplicate.

3-(4,5-Dimethylthiazol-2-yl)-5-(3-carboxymethoxyphenyl)-2-(4sulfophenyl)-2H-tetrazolium (MTS) viability assay. The viability of the cells was determiend by MTS assay (Sigma-Aldrich, 
A

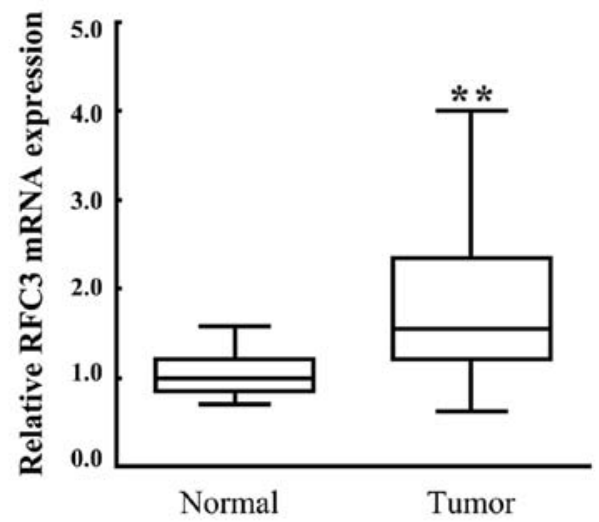

B

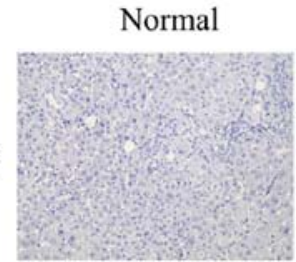

$\times 200$
Tumor
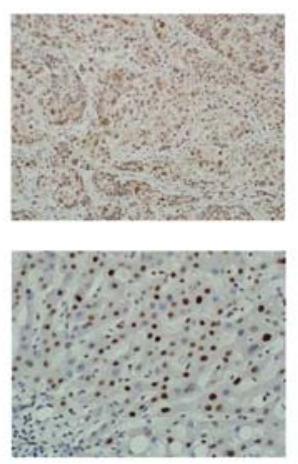

Figure 1. Replication factor C, subunit 3 (RFC3) is overexpressed in hepatocellular carcinoma (HCC) tissues. (A) RFC3 mRNA expression levels in liver tumor tissue samples and paired adjacent normal tissue samples ( 24 cases) were determined by RT-qPCR. GAPDH was used as an internal quantitative control. Three independent experiments were performed, and data are presented as the means \pm SD. ${ }^{* *} \mathrm{P}=0.0003$. (B) Immunohistochemical analysis of RFC3 protein expression in normal liver tissue samples (left panels) and HCC samples (right panels) treated with specific anti-RFC 3 antibody. Representative images were taken at x100 and x200 magnification.

St. Louis, MO, USA). Cells in the logarithmic growth phase were collected and seeded at a density of $1 \times 10^{3}$ cells/well in 96-well plates, in triplicate. On days 1,2, 3 and 4, MTS reagent was added to the cells at a ratio of 1:10 followed by incubation at $37^{\circ} \mathrm{C}$ for $4 \mathrm{~h}$. The solution was removed, and the cells were dissolved with dimethyl sulfoxide (Sigma-Aldrich). The absorbance of each well was measured using an LW R96 ELISA microplate reader (Diatek, West Bengal, India) at a wavelength of $490 \mathrm{~nm}$. Each experiment was performed in triplicate.

Cell growth curves. The cells were digested, and the number of living cells was counted using the method described by Freshney (17). The cells were then seeded in 3 wells of a 12 -well plate at approximately $1 \times 10^{5}$ cells/well. The living cells were digested and counted on days 1,2 and 3. The experiments were repeated 3 times, and averages were used to plot the cell growth curves.

Flow cytometric analysis. The cells were harvested and washed in PBS, and fixed in ice-cold $70 \%$ ethanol for $1 \mathrm{~h}$. Following treatment with RNase A (50 $\mu \mathrm{g} / \mathrm{ml}$; Sigma-Aldrich), the cells were stained with propidium iodide (PI; Sigma-Aldrich) for $30 \mathrm{~min}$ at room temperature and then analyzed and recorded using a FACSCalibur flow cytometer (BD Biosciences, San Jose, CA, USA). Cell cycle analysis was performed using FlowJo software (TreeStar Inc., Ashland, OR, USA).

Statistical analysis. SPSS 18.0 statistical software was used for statistical analysis. Data are presented as the means \pm SD, and all experiments were performed in triplicate $(n=3)$. Statistical analysis was performed using analysis of variance (ANOVA). A P-value $<0.05$ was considered to indicate a statistically significant difference.

\section{Results}

RFC3 is overexpressed in human liver tumor tissue. It has previously been reported that RFC3 has oncogenic activity and is overexpressed in epithelial carcinomas $(12,13)$. In this study, in order to determine whether the overexpression of RFC3 is associated with the development of HCC, liver tumor tissue samples from 24 patients were examined by RT-qPCR using RFC3-specific primers. Paired adjacent normal tissue samples were used as the controls. As shown in Fig. 1A, the mRNA expression level of RFC3 in tumor tissue samples was markedly upregulated compared with the adjacent non-tumor tissues. Moreover, IHC analysis revealed that strong positive staining in the liver tumor tissues, indicating the overexpression of RFC3 protein (Fig. 1B; compare 'Tumor' to 'Normal'). Taken together, these results indicated that RFC3 was upregulated in the liver tumor tissues.

RFC3 is overexpressed in HCC cell lines. To further confirm the stimulatory effect of RFC3 on HCC, we sought to identify an RFC3-sensitive cell line. For this purpose, 5 HCC cell lines (HepG2, BEL-7402, Hep3B, SMMC-7721 and LM3) were used to measure the mRNA and protein expression of RFC3 by RT-qPCR and western blot analysis, respectively. A normal hepatocyte cell line (L02) was used as the negative control. In brief, we found that both the mRNA and protein levels of RFC3 were increased in all HCC cell lines compared to the hepatocyte cell line, further confirming that RFC3 overexpression is associated with HCC (Fig. 2). Of the HCC cell lines, the SMMC-7721 cells exhibited the highest mRNA and protein expression of RFC3 and were thus used in subsequent experiments.

Downregulation of RFC3 through lentivirus-mediated shRNA in the SMMC-7721 cell line. To examine the role RFC3 plays in HCC, a stable HCC cell line in which RFC3 was knocked down was established using lentivirus-mediated RNA interference (RNAi) technology. The SMMC-7721 cell line was selected to establish the HCC cell line in which RFC3 would be knocked down. The knockdown effect was evaluated by RT-qPCR and western blot analysis. As shown in Fig. 3A, RFC3 mRNA expression was reduced by approximately $70 \%$ in the SMMC-7721-shRFC3 cells compared to the NC cells $(\mathrm{P}<0.01)$. Western blot analysis further confirmed that 


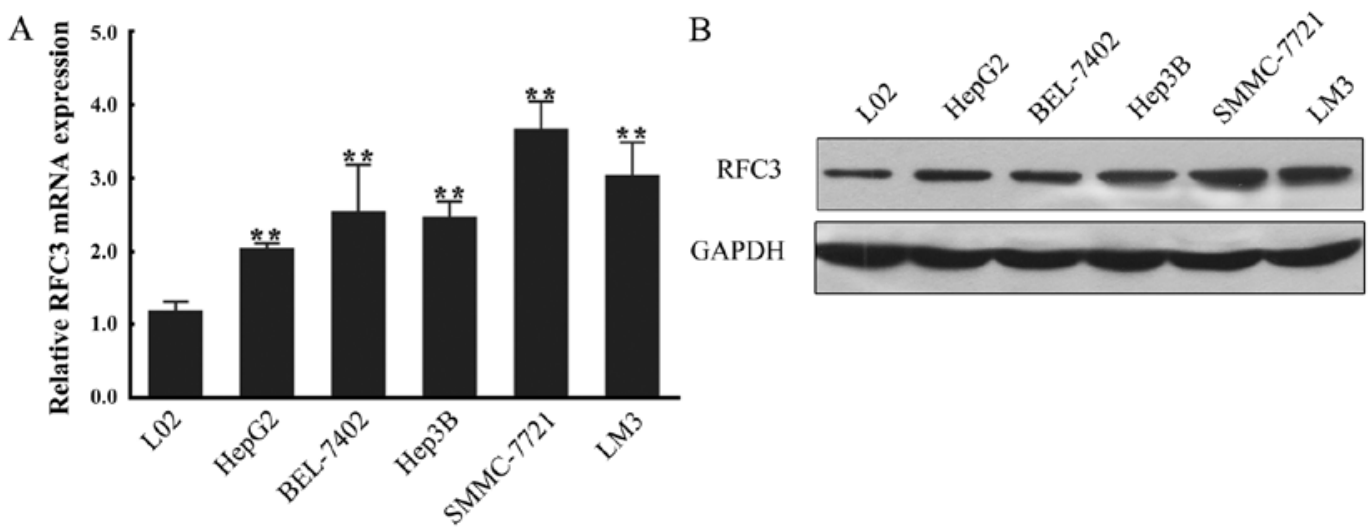

Figure 2. Replication factor C, subunit 3 (RFC3) is overexpressed in hepatocellular carcinoma (HCC) cells. (A) RFC3 mRNA expression was detected by RT-qPCR in human hepatocyte cells (L02) and 5 human HCC cell lines (HepG2, BEL-7402, Hep3B, SMMC-7721 and LM3) as indicated. (B) Western blot analysis was performed to determined RFC3 protein expression levels in the 6 cell lines, as described above. GAPDH was used as an internal quantitative control. Three independent experiments were performed, and data are presented as the means $\pm \mathrm{SD} .{ }^{* *} \mathrm{P}<0.01$.
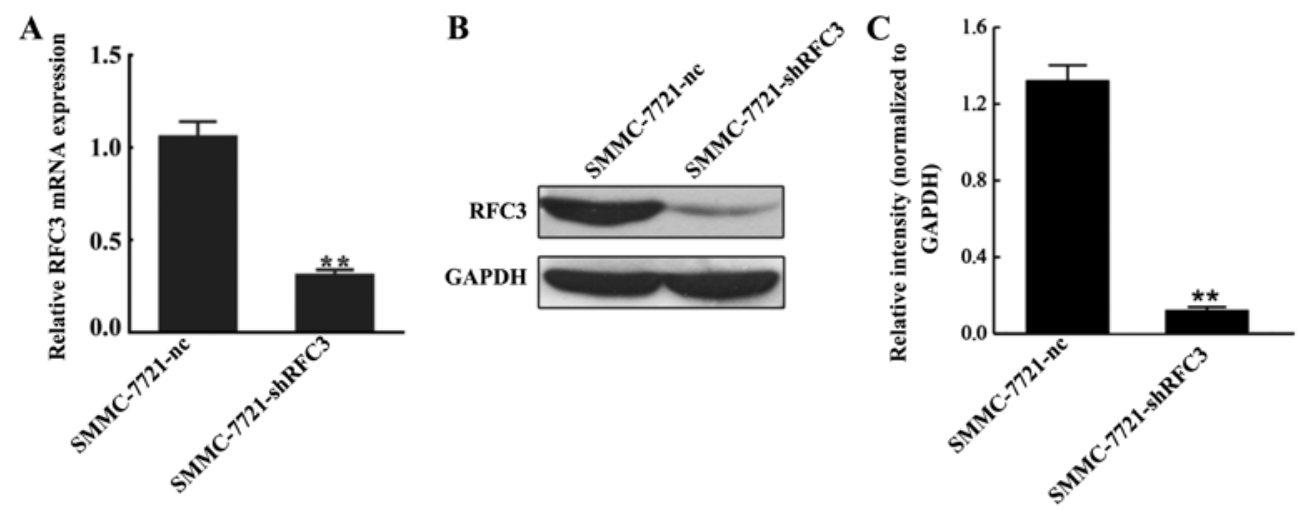

Figure 3. Downregulation of replication factor C, subunit 3 (RFC3) through lentivirus-mediated shRNA in the SMMC-7721 cell line. (A) RFC3 protein expression levels in SMMC-7721-nc (negative control) cells and SMMC-7721-shRFC3 cells were detected by western blot analysis. GAPDH is shown as the internal control. (B) mRNA levels of RFC3 in control cells and RFC3-silenced cells were measured by RT-qPCR. GAPDH was used as an internal quantitative control. Data are presented as the means \pm SD from 3 independent experiments. ${ }^{* *} \mathrm{P}<0.01$. SMMC-7721-shRFC3, cells in which RFC3 was knocked down by shRNA; SMMC-7721-nc, cells transfected with the negative control vector.

almost $90 \%$ of RFC3 expression was markedly suppressed in the SMMC-7721-shRFC3 cells (Fig. 3B and C).

Knockdown of RFC3 inhibits HCC cell proliferation and viability. We sought to examine the effects of RFC3 knockdown on HCC cells. To this end, we examined the proliferation and viability of SMMC-7721-shRFC3 cells using a cell colony formation assay, MTS viability assay and cell growth curve assay, respectively. As shown in Fig. 4A, statistical analysis indicated that the colony-forming ability of the SMMC-7721shRFC3 cells decreased to $62 \%(\mathrm{P}<0.01)$ compared with that of the NC cells which was $97 \%$. Moreover, a marked decrease in cellular viability was observed in the cells in which RFC3 was knocked down (SMMC-7721-shRFC3 cells). Furthermore, the cell growth curve assay revealed that the population of SMMC-7721-shRFC3 living cells was considerably lower compared with the NC cells (Fig. 4C). Collectively, these data indicated that the knockdown of RFC3 inhibited HCC cell proliferation and viability.

Knockdown of RFC3 induces HCC cell cycle arrest at the $S$ phase. As abnormal cell proliferation is closely associated with the dysregulation of the cell cycle (18), we examined whether the knockdown of RFC3 affects the HCC cell cycle using flow cytometric analysis. As shown in Fig. 5A and B, the knockdown of RFC3 significantly increased the percentage of cells in the $\mathrm{S}$ phase, but decreased that in the G0/G1 phase; moreover, the downregulation of RFC3 did not significantly alter the percentage of cells in the G2/M phase, indicating that the cell cycle was arrested at the $\mathrm{S}$ phase, when RFC3 was knocked down.

To further elucidate the mechanisms behind the cell cycle arrest at the S phase following the knockdown of RFC3, we measured the expression levels of cell cycle-related proteins by western blot analysis. As shown in Fig. 5C, in the HCC cells in which RFC3 was knocked down, the tumor suppressor genes, p53, p21 and p57 were all upregulated. In the cell cycle, p21 functions as a negative regulator that inhibits DNA synthesis and arrests the cell cycle at the G1/S phase by binding to and inhibiting the activity of cyclin-dependent kinase (CDK)2, CDK1, CDK4 and CDK6 complexes (19). p53 upregulates the expression of p21 (20). p57 is also a tight-binding inhibitor of CDK2, CDK4 and CDK6 complexes and a negative regulator of cell proliferation (21). Based on this information, our data indicated that RFC3 knockdown upregulated p53 expression, subsequently inducing the upregulation of $\mathrm{p} 21$, and eventually inhibiting the cell cycle. Similarly, the knockdown of 
A

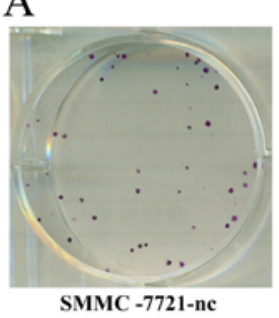

SMMC -7721-ne
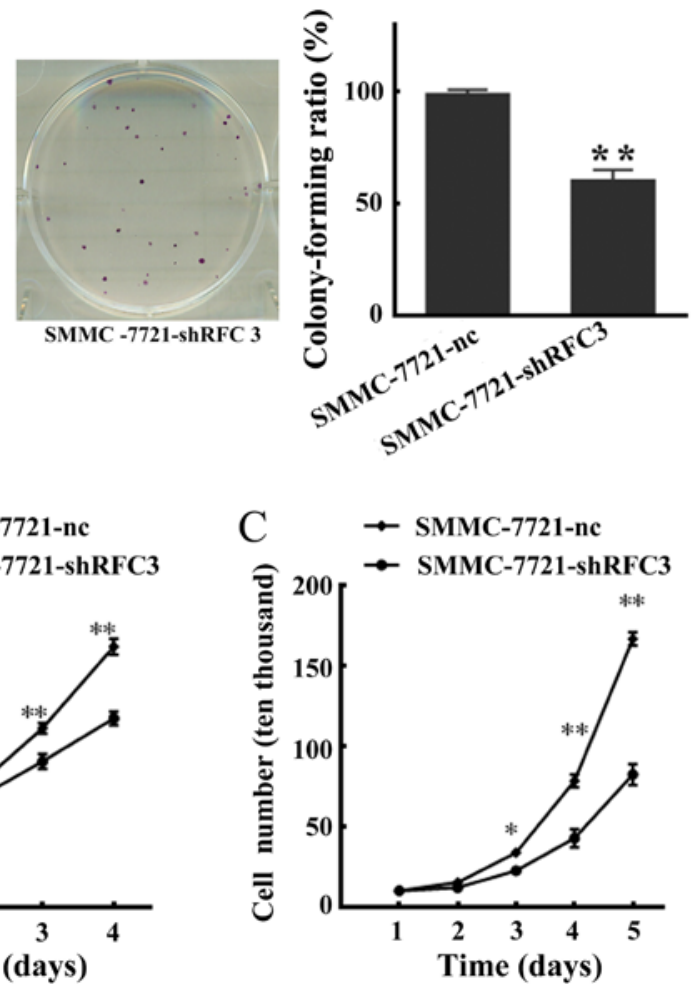

Figure 4. Downregulation of replication factor C, subunit 3 (RFC3) inhibits hepatocellular carcinoma (HCC) cell proliferation and viability. (A) Representative images of a single colony in plates are shown; downregulation of RFC3 inhibited the colony-forming ability of the cells. (B) Proliferation rates were determined by MTS assay on days 1-4 in the cells in which RFC3 was knocked down. (C) Effect of RFC3 silencing on the viability of SMMC-7721-shRFC3 cells was evaluated by cell growth curve assay. Data were obtained from 3 separate experiments, and each bar represents the mean \pm SD. ${ }^{* *} \mathrm{P}<0.01$. SMMC-7721-shRFC3, cells in which RFC3 was knocked down by shRNA; SMMC-7721-nc, cells transfected with the negative control vector.

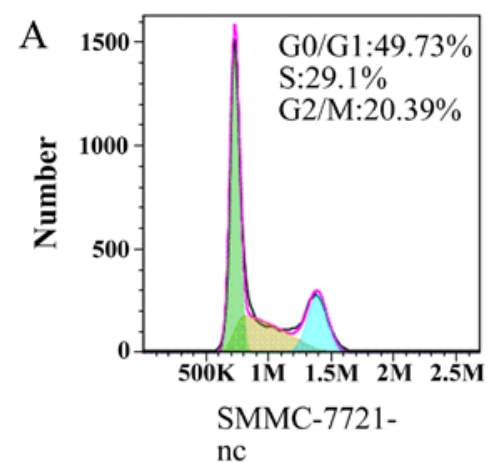

nc

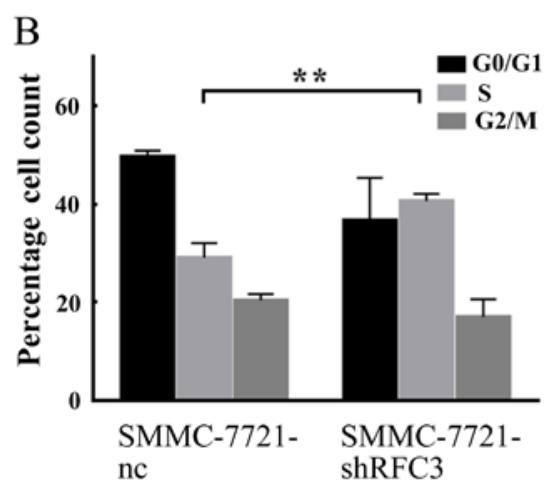

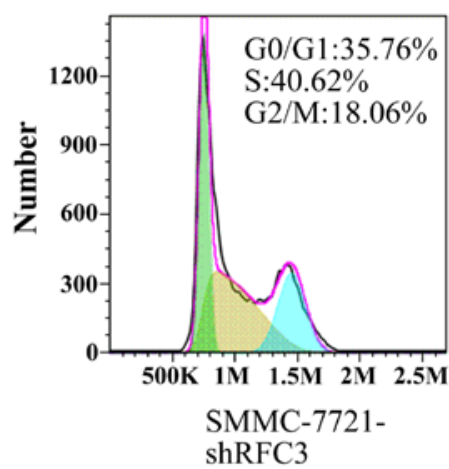

C

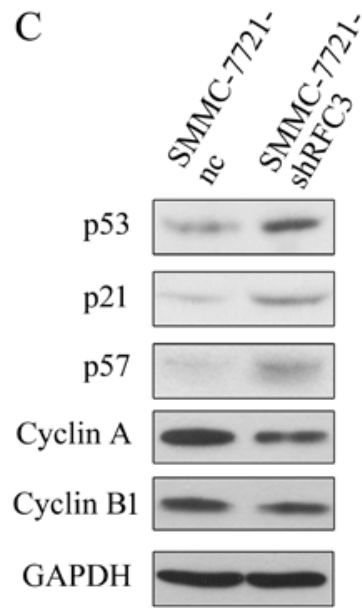

Figure 5. Suppression of replication factor C, subunit 3 (RFC3)-arrested hepatoma cells at S phase. (A) Cell cycle distribution of SMMC-7721-nc cells (negative control) (left panel) and SMMC-7721-shRFC3 cells (right panel) during the cell cycle were analyzed by FACS. Green, G0/G1 phase cells; yellow, S phase cells; blue, G2/M phase cells. (B) Knockdown of RFC3 significantly increased the percentage of cells in S phase and decreased the percentage of cells in G0/G1 phase. Data represent the means \pm SD of 3 independent experiments. (C) Western blot analysis of the cell cycle-related proteins. GAPDH was used as a loading control in all experiments. SMMC-7721-shRFC3, cells in which RFC3 was knocked down by shRNA; SMMC-7721-nc, cells transfected with the negative control vector. 
RFC3 upregulated p57 and directly resulted in the blocking of CDK complex activity. Indeed, the expression of cyclin A, a known cell cycle protein which is associated with the CDK2 complex required for G1-S phase transition (22), was downregulated (Fig. 5C). Of note, the expression of cyclin B1, a protein required for G2-M phase transition (23), was not markedly affected (Fig. 5C), suggesting that the knockdown of RFC3 specifically regulates G1-S phase transition. Taken together, these findings demonstrate that the knockdown of RFC3 induces $\mathrm{HCC}$ cell cycle arrest at the $\mathrm{S}$ phase by regulating tumor suppressor genes involved in G1-S phase transition.

\section{Discussion}

HCC is one of the most common and lethal malignancies in the world $(1,2)$. The identification of novel therapeutic targets that will contribute to the development and progression of $\mathrm{HCC}$ is obviously desirable if we are to combat this lethal disease. RFC3 is clearly one of the most important cancer antigens since it plays an indispensable role in DNA replication $(7,8,24,25)$. Previous studies have shown that the overexpression of RFC3 is closely related to esophageal adenocarcinomas and ovarian carcinomas, implying that this gene plays a role in tumor development $(12,13)$; however, its role in the development of HCC remains unclear. In the present study, we aimed to investigate the expression and biological functions of RFC3 in HCC tissues and cells.

The RFC complex has been identified as an important component of the cell cycle (10). The overexpression of the RFC complex was has been found to be responsible for DNA replication, DNA damage repair, checkpoints and inducing tumor formation $(7,10,12,25)$. Previous studies have demonstrated that RFC subunits are upregulated in different types of malignancies: RFC2, RFC3, RFC4 and RFC5 are upregulated in nasopharyngeal (13), ovarian (12), HCC (26) and human papillomavirus-positive squamous cell carcinomas (27), respectively. In agreement with these findings, we found that the expression of RFC3 was significantly upregulated in human HCC tissues and cell lines. Owing to the importance of RFC3 in the formation of DNA replication complex, it has been suggested that the overexpression of RFC3 is responsible for inducing tumor formation (13). Based on this hypothesis, RFC3 can be identified as one of the most important cancer antigens.

After noting that RCF3 was associated with HCC, we focused on whether the downregulation of RFC3 affects HCC cells. Lentivirus-mediated RNAi methods provide an attractive approach to efficiently suppress gene expression. The RNAi knockdown assays revealed that the suppression of RFC3 expression in HCC cells led to a considerable suppression of HCC cell viability and proliferation. These results are consistent with those of a previous study which demonstrated that RFC3 was overexpressed in esophageal adenocarcinoma and that RFC3 knockdown had an anti-proliferative effect (13). This suppression may be partly due to the induction of cancer cell cycle arrest at the $\mathrm{S}$ phase, the checkpoint of which is activated upon the formation and function of DNA replication complexes $(28,29)$. Given that RFC3 is one of the key components of DNA replication complexes, the downregulation of RFC3 is likely to result in the blockade of DNA replication complex formation and eventually suppress DNA replication.
The knockdown of RFC3 increased the levels of S phaseassociated proteins, such as p21, p53 and p57, but reduced the expression of cyclin A. In the cell cycle, the CDK2/cyclin A complex leads to progression through the G1-S phase transition, a step that is strictly regulated in the process of cell proliferation. p21 and p51,CDK inhibitors, bind to CDK2 and inhibit its activity. The overexpression of $\mathrm{p} 21$ and/or p51 results in cells remaining in the G1/S phase and the arrest of cell cycle progression. p53 is known to be an activator of p21 expression (20). It was proposed herein that the knockdown of RFC3 upregulates p53 expression, and subsequently induces p21 and/or p51 upregulation, and eventually inhibits G1-S phase transition. Further studies are required however, to focus on the detailed mechanisms behind the RFC3 regulation of cell cycle-related proteins.

In conclusion, the present study demonstrated that RFC3 was notably upregulated in HCC tissues and cell lines. The downregulation of RFC3 suppressed HCC cell viability and proliferation. Further experiments demonstrated that the knockdown of RFC3 induced HCC cell cycle arrest at the $\mathrm{S}$ phase. Tumor suppression was likely accomplished partially by inducing $S$ phase arrest and regulating cell cycle-related proteins. These results indicate that RFC3 plays an important role in the development of HCC. Therefore, we suggest that a specific enzymatic inhibitor to RFC3 may have therapeutic significance in the treatment of HCC.

\section{Acknowledgements}

This study was supported by grants from the National Natural Science Foundation of China (No. 8157111144) and the Science and Technology Planning Project of Guangzhou, Guangdong Province, China (No. 1563000226).

\section{References}

1. Slotta JE, Kollmar O, Ellenrieder V, Ghadimi BM and Homayounfar K: Hepatocellular carcinoma: Surgeon's view on latest findings and future perspectives. World J Hepatol 7: 1168-1183, 2015.

2. Huang CY, Lin CS, Tai WT, Hsieh CY, Shiau CW, Cheng AL and Chen KF: Sorafenib enhances radiation-induced apoptosis in hepatocellular carcinoma by inhibiting STAT3. Int J Radiat Oncol Biol Phys 86: 456-462, 2013.

3. McGlynn KA, Petrick JL and London WT: Global epidemiology of hepatocellular carcinoma: an emphasis on demographic and regional variability. Clin Liver Dis 19: 223-238, 2015.

4. Yang HI, Lee MH, Liu J and Chen CJ: Risk calculators for hepatocellular carcinoma in patients affected with chronic hepatitis B in Asia. World J Gastroenterol 20: 6244-6251, 2014.

5. Bahri O, Ezzikouri S, Alaya-Bouafif NB, Iguer F, Feydi AE, Mestiri H, Benazzouz M, Khalfallah T, Afifi R, Elkihal L, et al: First multicenter study for risk factors for hepatocellular carcinoma development in North Africa. World J Hepatol 3: 24-30, 2011.

6. Culligan KM and Hays JB: DNA mismatch repair in plants. An Arabidopsis thaliana gene that predicts a protein belonging to the MSH2 subfamily of eukaryotic MutS homologs. Plant Physiol 115: 833-839, 1997.

7. Pascucci B, Stucki M, Jónsson ZO, Dogliotti E and Hübscher U: Long patch base excision repair with purified human proteins. DNA ligase I as patch size mediator for DNA polymerases delta and epsilon. J Biol Chem 274: 33696-33702, 1999.

8. Shimada M, Okuzaki D, Tanaka S, Tougan T, Tamai KK, Shimoda C and Nojima H: Replication factor C3 of Schizosaccharomyces pombe, a small subunit of replication factor $\mathrm{C}$ complex, plays a role in both replication and damage checkpoints. Mol Biol Cell 10: 3991-4003, 1999. 
9. Xia ST, Xiao LT, Bi DL and Zhu ZH: Arabidopsis replication factor $\mathrm{C}$ subunit 1 plays an important role in embryogenesis. Zhi Wu Sheng Li Yu Fen Zi Sheng Wu Xue Xue Bao 33: 179-187, 2007.

10. Sancar A, Lindsey-Boltz LA, Unsal-Kaçmaz K and Linn S: Molecular mechanisms of mammalian DNA repair and the DNA damage checkpoints. Annu Rev Biochem 73: 39-85, 2004.

11. Mossi R and Hübscher U: Clamping down on clamps and clamp loaders - the eukaryotic replication factor C. Eur J Biochem 254: 209-216, 1998.

12. Shen H, Cai M, Zhao S, Wang H, Li M, Yao S and Jiang N: Overexpression of RFC3 is correlated with ovarian tumor development and poor prognosis. Tumour Biol 35: 10259-10266, 2014.

13. Xiong S, Wang Q, Zheng L, Gao F and Li J: Identification of candidate molecular markers of nasopharyngeal carcinoma by tissue microarray and in situ hybridization. Med Oncol 28 (Suppl 1): S341-S348, 2011.

14. Xia S, Xiao L, Gannon P and Li X: RFC3 regulates cell proliferation and pathogen resistance in Arabidopsis. Plant Signal Behav 5: 168-170, 2010.

15. Maeng S, Kim GJ, Choi EJ, Yang HO, Lee DS and Sohn YC: 9-Cis-retinoic acid induces growth inhibition in retinoid-sensitive breast cancer and sea urchin embryonic cells via retinoid $X$ receptor $\alpha$ and replication factor C3. Mol Endocrinol 26: 1821-1835, 2012.

16. Hu K, Wang J, Yao Z, Liu B, Lin Y, Liu L and Xu L: Expression of cytoskeleton regulatory protein Mena in human hepatocellular carcinoma and its prognostic significance. Med Oncol 31: 939, 2014.

17. Freshney RI, Sherry A, Hassanzadah M, Freshney M, Crilly P and Morgan D: Control of cell proliferation in human glioma by glucocorticoids. Br J Cancer 41: 857-866, 1980.

18. Wang J, Gong L, Zhu SJ, Zhu Q, Yao L, Han XJ, Zhang JR, Li YH and Zhang W: The human homolog of Drosophila headcase acts as a tumor suppressor through its blocking effect on the cell cycle in hepatocellular carcinoma. PLoS One 10: e137579, 2015.

19. Gartel AL and Radhakrishnan SK: Lost in transcription: p21 repression, mechanisms, and consequences. Cancer Res 65: 3980-3985, 2005.
20. el-Deiry WS, Tokino T, Velculescu VE, Levy DB, Parsons R, Trent JM, Lin D, Mercer WE, Kinzler KW and Vogelstein B: WAF1, a potential mediator of p53 tumor suppression. Cell 75: 817-825, 1993.

21. Lee MH, Reynisdóttir I and Massagué J: Cloning of p57KIP2, a cyclin-dependent kinase inhibitor with unique domain structure and tissue distribution. Genes Dev 9: 639-649, 1995.

22. Guadagno TM and Newport JW: Cdk2 kinase is required for entry into mitosis as a positive regulator of Cdc2-cyclin B kinase activity. Cell 84: 73-82, 1996.

23. De Souza CP, Ellem KA and Gabrielli BG: Centrosomal and cytoplasmic Cdc2/cyclin B1 activation precedes nuclear mitotic events. Exp Cell Res 257: 11-21, 2000.

24. Johnson A, Yao NY, Bowman GD, Kuriyan J and O'Donnell M: The replication factor $\mathrm{C}$ clamp loader requires arginine finger sensors to drive DNA binding and proliferating cell nuclear antigen loading. J Biol Chem 281: 35531-35543, 2006.

25. Li X and Burgers PM: Molecular cloning and expression of the Saccharomyces cerevisiae RFC3 gene, an essential component of replication factor C. Proc Natl Acad Sci USA 91: 868-872, 1994.

26. Arai M, Kondoh N, Imazeki N, Hada A, Hatsuse K, Matsubara O and Yamamoto M: The knockdown of endogenous replication factor $\mathrm{C} 4$ decreases the growth and enhances the chemosensitivity of hepatocellular carcinoma cells. Liver Int 29: 55-62, 2009.

27. Martinez I, Wang J, Hobson KF, Ferris RL and Khan SA: Identification of differentially expressed genes in HPV-positive and HPV-negative oropharyngeal squamous cell carcinomas. Eur J Cancer 43: 415-432, 2007.

28. Koch HB, Zhang R, Verdoodt B, Bailey A, Zhang CD, Yates JR III, Menssen A and Hermeking H: Large-scale identification of c-MYC-associated proteins using a combined TAP/MudPIT approach. Cell Cycle 6: 205-217, 2007.

29. Green CM, Erdjument-Bromage H, Tempst P and Lowndes NF: A novel Rad 24 checkpoint protein complex closely related to replication factor C. Curr Biol 10: 39-42, 2000. 